\title{
Complete closure versus simple closure for perforations during colorectal endoscopic submucosal dissection
}

\section{다 (1) $\odot$}

Authors

Satoshi Kinoshita ${ }^{1}$, Toshihiro Nishizawa ${ }^{1,2}$, Ai Fujimoto ${ }^{1}$, Hideki Mori ${ }^{1}$, Yuichiro Hirai ${ }^{1}$, Yoshihiro Nakazato ${ }^{1}$, Masahiro Kikuchi ${ }^{1}$, Toshio Uraoka ${ }^{3}$

Institutions

1 Department of Gastroenterology, National Hospital Organization Tokyo Medical Center, Gunma University, Tokyo, Japan

2 Department of Gastroenterology and Hepatology, International University of Health and Welfare Mita Hospital, Gunma University, Tokyo, Japan

3 Department of Gastroenterology and Hepatology, Gunma University, Tokyo, Japan

submitted 6.6.2019

accepted after revision 5.8.2019

Bibliography

DOI https://doi.org/10.1055/a-1012-1838 |

Endoscopy International Open 2020; 08: E76-E80

(c) Georg Thieme Verlag KG Stuttgart · New York

eISSN 2196-9736

Corresponding author

Toshihiro Nishizawa, MD, PhD, Department of

Gastroenterology and Hepatology, International University of Health and Welfare Mita Hospital, Mita 1-4-3 Minato-ku, Tokyo, $180-8392$

Fax: +81-3-5363-3895

nisizawa@kf7.so-net.jp

\section{ABSTRACT}

Background and study aims The efficacy of complete closure versus simple closure for perforations during endoscopic submucosal dissection (ESD) has never been evaluated. We evaluated the efficacy of complete closure and simple closure for perforations and muscular layer injuries incurred during ESD.

Patients and methods Thirty-four consecutive patients who underwent "complete closure" or "simple closure" for correction of perforations and muscular layer injuries during colorectal ESD were enrolled in this study. Complete closure was performed by the mucosa-submucosa clip closure method using only conventional endo-clips. For simple closure, endo-clips are placed just for perforation or muscular layer injury, while leaving any mucosal defects open. Results Among the 15 patients in the complete closure group, eight developed perforations and seven developed muscular layer injuries. Among the 19 patients in the simple closure group, six developed perforations and 13 developed muscular layer injuries during the ESD procedure. There were no statistically significant differences between the two groups in inflammatory reactions, adverse events, or length of the hospital stay.

Conclusion Complete closure and simple closure for perforations and muscular layer injuries during ESD seem to have similar efficacy. While simple closure for a perforation during ESD seems sufficient, further study will be required to confirm our results.

\section{Introduction}

Endoscopic submucosal dissection (ESD) has recently been gaining acceptance as an alternative to endoscopic mucosal resection (EMR). ESD can provide en bloc complete resection of gastrointestinal mucosal lesions of any size [1-3]. Perforation is one of the most serious adverse events (AEs) associated with colorectal ESD. Although rates of perforation have been decreasing through improvements in techniques and equipment, perforation still occurs in $1.4 \%$ to $10.0 \%$ of all colorectal ESDs performed [4-7].
A perforation occurring during an ESD procedure must be clipped [8]. If a perforation can be successfully closed by endoscopic clipping during ESD, the patient can be managed conservatively thereafter. Endoscopic clipping is usually a simple closure performed by placing endo-clips to close a perforation. No closure of mucosal defects is attempted during the procedure. Complete closure is a more complicated procedure that is performed using various methods. The methods for complete closure reported in recent studies have included endoscopic purse-string suture [9], the slip knot clip suturing method [10, $11]$, the string clip suturing method [12], the "loop clip" method [13], "Hold-and-drag" closure [14,15], and the mucosa-sub- 
mucosa clip closure method [16]. Complete closure of a mucosal defect after ESD appears to be effective in preventing AEs. Efficacy of complete closure versus simple closure for a perforation during ESD, however, has never been previously evaluated. In the current study we evaluated efficacy of complete closure and simple closure for perforations and muscular layer injuries during ESD.

\section{Patents and methods}

\section{Patients}

One hundred sixty-five patients underwent colorectal ESD at Tokyo Medical Center between November 2016 and July 2019. Of them, 34 patients experienced perforations or muscular layer injuries during the ESD procedure ( $>$ Fig. 1, $>$ Fig. 2). A perforation was defined as a colonic mural defect ( $\triangleright$ Fig.2a). A muscular layer injury was defined as a defect of the muscular layer ( $\$$ Fig. $\mathbf{3}$ ).

During the initial period of the study, between November 2016 and April 2017, perforations and muscular layer injuries were closed by the simple closure method, while mucosal defects were left open. Between May 2017 and May 2018, mucosal defects $\leq 5 \mathrm{~cm}$ were completely closed by the mucosa-submucosa clip closure method. In the latter period of the study, between August 2018 and July 2019, the endoscopists performing the ESD arbitrarily chose between the simple closure method and complete closure method when closure was necessary. This study protocol was approved by the Institutional Review Board of Tokyo Medical Center (registration number: R17096). Written informed consent was obtained from all patients.

\section{Methods for complete closure and simple closure}

The mucosa-submucosa clip closure method was used for complete closure $[16,17]$. The endo-clip (EZ Clip ${ }^{\circledR}, \mathrm{HX}-610-090 \mathrm{~L}$ (long-type), OLYMPUS, Tokyo, Japan) was placed at the edge of each mucosal defect after colorectal ESD. The two arms of the endo-clip respectively gripped the mucosa and submucosa in a direction parallel to the short axis of the defect. Though the placement of the clip significantly reduced the size of the mucosal defect, additional endo-clips had to be placed on the two sides of the defect to achieve complete closure. Finally, endoscopic inspection was performed to visually confirm that the closure was complete ( $\triangleright$ Fig. $\mathbf{1}$ ).

Simple closure was performed by placing endo-clips just for perforations and muscular layer injuries, while leaving any mucosal defects open ( $>$ Fig. 2 ).

The procedures in this study were performed by four endoscopists, each of whom was considered an expert in ESD and had performed more than 50 colorectal ESD procedures before the study began. Trainee physicians often took part in the ESD procedures, always under the direct supervision of an expert staff physician.

\section{Evaluation of the procedure}

The following parameters were evaluated: size of the resected specimen, procedure time required to attain complete closure or simple closure, number of clips, blood test results (white

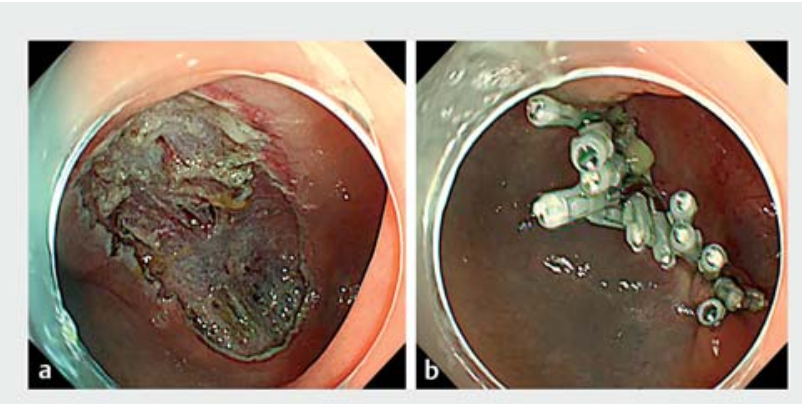

- Fig. 1 Complete closure. a Mucosal defect after colonic ESD. b Complete closure by the mucosa-submucosa clip closure method.

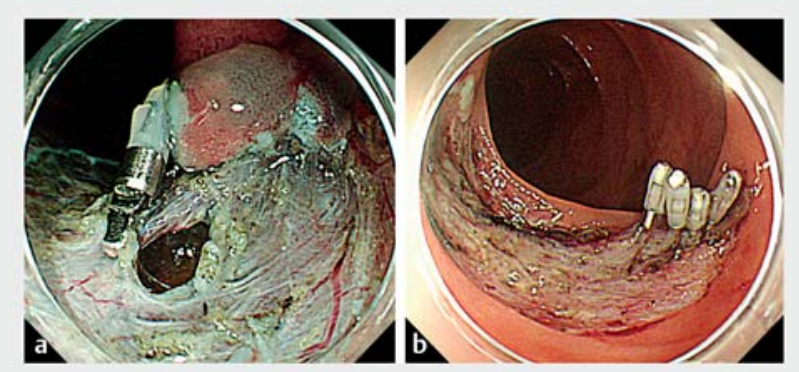

- Fig. 2 Simple closure. a Perforation during ESD. b Endo-clips were placed just for perforations.

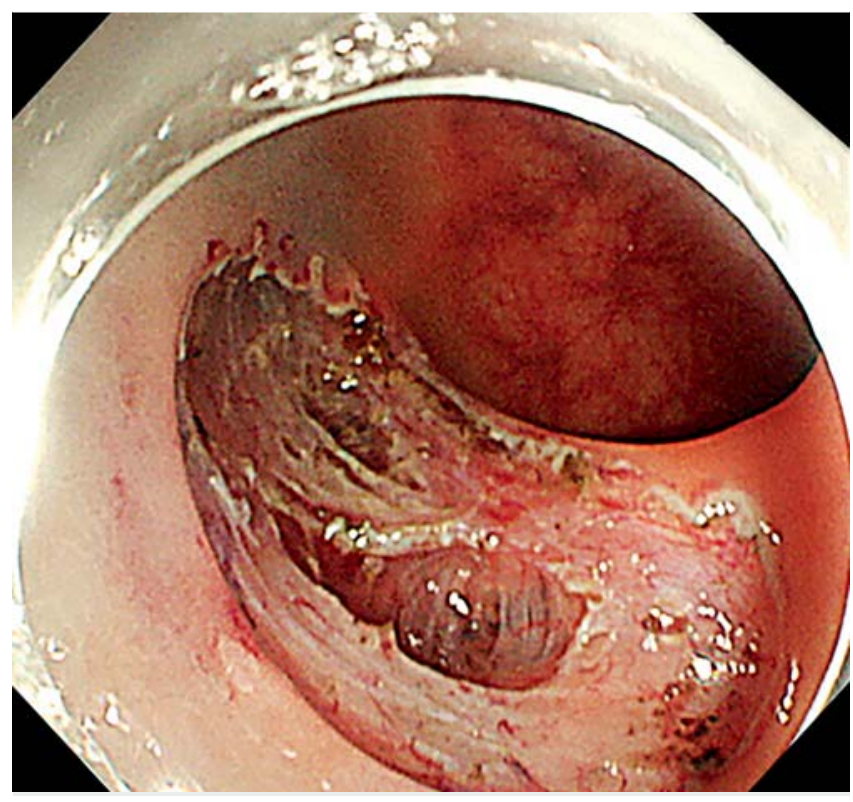

- Fig. 3 Representative image of a muscular layer injury.

blood cell count [WBC] and C-reactive protein) on the day after ESD, AEs (delayed perforation and delayed bleeding), abdominal pain, fever, emergency surgery, and length of hospital stay.

Procedure time was measured from insertion of the first clip to completion of the procedure. Delayed perforation was defined by presence of free air on postoperative $x$-ray, with severe 
- Table 1 Baseline patent characteristics.

\begin{tabular}{|c|c|c|c|}
\hline & $\begin{array}{l}\text { Complete } \\
\text { closure }\end{array}$ & $\begin{array}{l}\text { Simple } \\
\text { closure }\end{array}$ & $P$ value \\
\hline \multicolumn{4}{|l|}{ Patient } \\
\hline - Number & 15 & 19 & \\
\hline - Male:female & $8: 7$ & 9: 0 & 1 \\
\hline - Age & $65.8 \pm 11.1$ & $72.9 \pm 10.2$ & 0.06 \\
\hline \multicolumn{3}{|l|}{ Complications during ESD } & 0.353 \\
\hline - Perforation during ESD & 8 & 6 & \\
\hline - Muscular layer injury & 7 & 13 & \\
\hline \multicolumn{4}{|l|}{ Resected specimen } \\
\hline - Size & $31.6 \pm 8.65$ & $38 \pm 10.4$ & 0.06 \\
\hline \multicolumn{3}{|l|}{ Location } & 0.10 \\
\hline - Cecum & 1 & 4 & \\
\hline - Ascending colon & 4 & 3 & \\
\hline - Transverse colon & 4 & 4 & \\
\hline - Descending colon & 0 & 3 & \\
\hline - Sigmoid colon & 3 & 0 & \\
\hline - Rectum & 3 & 5 & \\
\hline \multicolumn{4}{|l|}{ Operator } \\
\hline - Experts (>50 ESD cases) & 8 & 12 & 0.82 \\
\hline $\begin{array}{l}\text { - Non-experts (<50 ESD } \\
\text { cases) }\end{array}$ & 7 & 7 & \\
\hline ESD, endoscopic submucosal d & ection. & & \\
\hline
\end{tabular}

abdominal pain in patients without intraoperative perforation. Delayed bleeding was defined as bleeding symptoms or hemoglobin loss ( $\geq 2 \mathrm{~g} / \mathrm{dL}$ ) [18]. Abdominal pain was defined as sustained spontaneous pain or regional rebound tenderness after ESD. Fever was defined as a body temperature of $\geq 38^{\circ} \mathrm{C}$ developing at any time from 4 hours to 3 days after ESD.

\section{Statistics}

All continuous variables were expressed as mean \pm standard deviation. Differences between the two groups were detected using the Student's t-test or Welch's $t$-test for continuous data. Categorical secondary outcomes were compared using the chi-squared test. $P<0.05$ was considered statistically significant. All statistical analyses were performed using Stat Mate IV software (ATOMS, Tokyo, Japan).

\section{Results}

Among the 34 patients, 15 patients underwent complete closure and 19 underwent simple closure. Table 1 summarizes characteristics and outcomes of the complete closure group and simple closure group. Among the 15 patients in the complete closure group, eight developed perforations and seven
- Table 2 Comparison of clinical courses and outcomes between complete closure and simple closure

\begin{tabular}{|l|l|l|l|}
\hline & $\begin{array}{l}\text { Complete } \\
\text { closure }\end{array}$ & $\begin{array}{l}\text { Simple } \\
\text { closure }\end{array}$ & P value \\
\hline Patient & \multicolumn{2}{|l|}{} \\
\hline - Number & 15 & 19 & \\
\hline Closure & & & \\
\hline - Procedure time (sec) & $752 \pm 277$ & $161 \pm 91.8$ & $<0.001$ \\
\hline - Number of clips & $9.73 \pm 2.89$ & $2.68 \pm 1.49$ & $<0.001$ \\
\hline Blood test & & & \\
\hline - WBC & $9220 \pm 2962$ & $8694 \pm 2508$ & 0.579 \\
\hline - C-reactive protein & $1.87 \pm 1.65$ & 2.612 .1 & 0.275 \\
\hline Adverse event & & & \\
\hline - Delayed perforation & 0 & 0 & - \\
\hline - Delayed bleeding & 0 & 0 & - \\
\hline - Abdominal pain & 3 & 9 & 0.195 \\
\hline - Fever & 7 & 8 & 0.935 \\
\hline Emergency surgery & 0 & $7.79 \pm 1.34$ & 0.205 \\
\hline Length of hospital stay & $7.2 \pm 1.48$ & & \\
\hline WBC, white blood count. & & & \\
\hline
\end{tabular}

developed muscular layer injuries during the ESD procedure. Among the 19 patients in the simple closure group, six developed perforations and 13 developed muscular layer injuries during the ESD procedure.

Mean procedure time for complete closure was $790 \pm 279$ sec ( $\bullet$ Table 2 ). The time for simple closure was significantly shorter than that for complete closure $(P<0.001)$. Mean number of clips was significantly lower in the simple closure group than in the complete closure group $(P<0.001)$. There were no significant differences between the two groups in the findings on WBC, C-reactive protein, delayed perforation, delayed bleeding, abdominal pain, fever, emergency surgery, or length of hospital stay.

\section{Discussion}

There were no statistically significant differences in inflammatory reactions, AEs, or length of hospital stay between the complete closure and simple closure procedures for correction of perforations and muscular layer injuries incurred during ESD. Considering the longer clipping time and cost of the clips required for complete closure, we propose that a simple closure may suffice.

Recent studies have reported that patients with colonic perforation can be managed conservatively with endoscopic clipping [19-21]. Hotta et al. obtained successful results by endoscopic clipping in 10 of 11 patients (90.9\%) with perforations 
[20]. Criteria for non-surgical treatment of a perforation caused by colonic ESD in their study were an absence of diffuse peritonitis and successful perforation closure [20]. Our study also showed that patients with colonic perforations treated by complete closure or simple closure could be managed conservatively.

Several studies have compared complete closure and nonclosure after colonic ESD [22-24]. A retrospective study by Fujihara et al. showed that complete closure significantly reduced inflammatory reactions and abdominal pain [22]. A randomized controlled trial (RCT) by Osada et al. found no significant differences between complete closure and non-closure for AEs, although complete closure accelerated wound healing at 4 weeks after ESD [24]. A recent meta-analysis of RCTs has compared efficacy of prophylactic clipping for colorectal endoscopic resection, including two RCTs with ESD cases [25]. Prophylactic clipping did not decrease occurrence of AEs after colorectal endoscopic resection. Theoretically, prophylactic clipping to avoid AEs may seem attractive and safe [26]. Data from available trials, however, are too scarce to prove this conjecture.

In monetary terms, the cost of one clip is approximately 788 yen (USD 7.9). In this study, a mean of 9.8 were placed in the complete closure group and a mean of 2.8 clips were placed in the simple closure group. Thus, 5516 yen (USD 55.3) could have been saved in every intervention in which a simple closure was performed in lieu of a complete closure.

This study had some limitations. First, the small number of patients was a limiting factor. This study may have lacked statistical power and may have failed to detect unrevealed but statistically important differences. While the current data suggested that complete closure reduced abdominal pain and the length of the hospital stay, they were not definitive. Second, the study was a retrospective review of patients treated at a single institution. Third, background differences may have introduced bias between the simple closure and complete closure groups, as the patients were not randomized to the two groups. Complete closure of a large mucosal defect is technically difficult, hence the differences between the groups indicate a significant selection bias. Furthermore, many of the simple closures performed were for muscular layer injuries. A muscular layer injury can be managed more easily than a perforation, which improved the outcome in the simple closure group. A prospective randomized study directly comparing a complete closure group with a simple closure group would have had advantages.

\section{Conclusion}

In conclusion, complete closure and simple closure for perforations and muscular layer injuries during ESD seem to have similar efficacy. Although simple closure for perforation during ESD might suffice, further study is required to confirm our results.

\section{Acknowledgements}

This study was supported by a grant from the National Hospital Organization (No. H30-NHO [gastroenterology], to H.M.).
Competing interests

None

References

[1] Nishizawa T, Yahagi N. Endoscopic mucosal resection and endoscopic submucosal dissection: technique and new directions. Curr Opin Gastroenterol 2017; 33: 315-319

[2] Pioche M, Rivory J, Nishizawa T et al. Randomized comparative evaluation of endoscopic submucosal dissection self-learning software in France and Japan. Endoscopy 2016; 48: 1076-1083

[3] Kinoshita S, Uraoka T, Nishizawa T et al. The role of colorectal endoscopic submucosal dissection in patients with ulcerative colitis. Gastrointest Endosc 2018; 87: 1079-1084

[4] Saito Y, Uraoka T, Matsuda T et al. Endoscopic treatment of large superficial colorectal tumors: a case series of 200 endoscopic submucosal dissections (with video). Gastrointest Endosc 2007; 66: 966973

[5] Fujishiro M, Yahagi N, Nakamura M et al. Endoscopic submucosal dissection for rectal epithelial neoplasia. Endoscopy 2006; 38: 493-497

[6] Byeon JS. Colonic perforation: can we manage it endoscopically? Clin Endosc 2013; 46: 495-499

[7] Kaosombatwattana U, Yamamura T, Limsrivilai ] et al. Preoperative endoscopic predictors of severe submucosal fibrosis in colorectal tumors undergoing endoscopic submucosal dissection. Endosc Int Open 2019; 7: E421-E430

[8] Tanaka S, Kashida H, Saito Y et al. JGES guidelines for colorectal endoscopic submucosal dissection/endoscopic mucosal resection. Dig Endosc 2015; 27: 417-434

[9] Matsuda T, Fujii T, Emura F et al. Complete closure of a large defect after EMR of a lateral spreading colorectal tumor when using a twochannel colonoscope. Gastrointest Endosc 2004; 60: 836-838

[10] Nishizawa T, Uraoka T, Suzuki H et al. New endoscopic suturing method: slip knot clip suturing. Gastrointest Endosc 2015; 82: $570-$ 571

[11] Nishizawa T, Suzuki H, Arano T et al. Characteristics of gastric cancer detected within 1 year after successful eradication of Helicobacter pylori. J Clin Biochem Nutr 2016; 59: 226-230

[12] Yahagi N, Nishizawa T, Akimoto T et al. New endoscopic suturing method: string clip suturing method. Gastrointest Endosc 2016; 84: 1064-1065

[13] Osada T, Sakamoto N, Ritsuno H et al. Process of wound healing of large mucosal defect areas that were sutured by using a loop clip-assisted closure technique after endoscopic submucosal dissection of a colorectal tumor. Gastrointest Endosc 2013; 78: 793-798

[14] Akimoto T, Goto O, Sasaki M et al. "Hold-and-drag" closure technique using repositionable clips for large mucosal defects after colonic endoscopic submucosal dissection. Endosc Int Open 2016; 4: E1068E1072

[15] Akimoto T, Goto O, Nishizawa T et al. Endoscopic closure after intraluminal surgery. Dig Endosc 2017; 29: 547-558

[16] Banno S, Nishizawa T, Kinoshita S et al. Endoscopic mucosa-submucosal clip closure method. Endoscopy 2017; 49: E307-E308

[17] Nishizawa T, Banno S, Kinoshita $S$ et al. Feasibility of endoscopic mucosa-submucosa clip closure method (with video). Endosc Int Open 2018; 6: E1070-E1074

[18] Nishizawa T, Ochiai Y, Uraoka T et al. Endoscopic slip-knot clip suturing method: prospective pilot study (with video). Gastrointest Endosc 2017; 85: 433-437 
[19] Takamaru H, Saito Y, Yamada M et al. Clinical impact of endoscopic clip closure of perforations during endoscopic submucosal dissection for colorectal tumors. Gastrointest Endosc 2016; 84: 494-502 e491

[20] Hotta K, Shinohara T, Oyama T et al. Criteria for non-surgical treatment of perforation during colorectal endoscopic submucosal dissection. Digestion 2012; 85: 116-120

[21] Sunata Y, Nishizawa T, Kinoshita S et al. A lesson from inappropriate single closure of a perforation during endoscopic submucosal dissection. Endoscopy 2018; 50: E25-E26

[22] Fujihara S, Mori H, Kobara $\mathrm{H}$ et al. The efficacy and safety of prophylactic closure for a large mucosal defect after colorectal endoscopic submucosal dissection. Oncol Rep 2013; 30: 85-90
[23] Harada H, Suehiro S, Murakami D et al. Clinical impact of prophylactic clip closure of mucosal defects after colorectal endoscopic submucosal dissection. Endosc Int Open 2017; 5: E1165-E1171

[24] Osada T, Sakamoto N, Ritsuno H et al. Closure with clips to accelerate healing of mucosal defects caused by colorectal endoscopic submucosal dissection. Surg Endosc 2016; 30: 4438-4444

[25] Nishizawa T, Suzuki H, Goto O et al. Effect of prophylactic clipping in colorectal endoscopic resection: A meta-analysis of randomized controlled studies. United European Gastroenterol J 2017; 5: 859-867

[26] Nishizawa T, Akimoto T, Uraoka T et al. Endoscopic string clip suturing method: a prospective pilot study (with video). Gastrointest Endosc 2018; 87: 1074-1078 